\title{
Optimal Resource Allocation for Device -to-Device Communication and Minimizing the base Station Transmission Power
}

\author{
P. Karthik \\ ME-Embedded System \\ Technologies \\ SKP Engineering College \\ Tiruvannamalai, India
}

\author{
S. Priyanka \\ Research Scholar/Electronics \\ Engineering \\ VIT University \\ Vellore, India
}

\author{
G. Loganathan \\ ME- Communication Systems \\ Sri Venkateswara College Of \\ Engineering \\ Chennai, India
}

\begin{abstract}
The Long term Evolution (LTE) and LTE - Advanced (LTE A) are provides the new communications such has Device to Device Communications (D2D), cellular networks direct transmission between the two are more devices. In this paper, we consider a system for D2D communication in cellular networks and minimizing the transmission power in Base station (BS). The D2D systems followed spectrum sharing protocol and Multiuser MIMO-OFDMA transmission system base station follows three power saving mechanism: Power control, Discontinuous transmission and antenna adaptation. The power control using the algorithm is known about sleep mode duration and channel knowledge. A simulation result denotes the D2D transmission versus cellular transmission and improves the system performance by reusing the cellular resources.
\end{abstract}

\section{Keywords}

D2D Communication, power control, LTE, OFDMA, MIMO, spectrum sharing protocol.

\section{INTRODUCTION}

In last few years cellular communication is developed, mobile users are increased and also introduce new features of smart technologies such as virtual environment of data transfer using D2D communications. It performs high speed communication for local cellular networks in approaches [1][4] and to minimizing the BS power consumptions as in [5].The D2D communications proposed for different time slot and same frequency used and carefully managed interference, reducing Base station traffic and minimizing transmission power. The different power saving mechanism in Radio Resource Management (RRM), power control is very important for cellular networks and beneficial to link in adaptation, interference reduction. Depending off on time of day or geographic location BS may be an idle but mobile terminal not negatively affected. The power control mechanism is complex to minimizing transmission power for Multi-user Orthogonal frequency division Multiplexing (OFDM), Multiple-Input Multiple-Output (MIMO) is to consider for reducing the transmission power in cellular BS.

The optimal transmitter antenna is used for MIMO in depends on Signal to Noise Ratio (SNR). In another RRM energy saving mechanism is Discontinuous transmission (DTX) and antenna adaptation (AA), the AA is adapt in MIMO resource allocation problem. The D2D communication proposed spectrum sharing protocol for allows bi-directional communication for BS and cellular network in between relay is fixed. The Cognitive Radio network for un-allocated frequency use method, allocating cellular network for transmission for resource units. The LTE [6],[7] transmission power minimizing for following algorithm Resource allocation using Antenna adaptation, power control and sleep modes (RAPS).

The RAPS algorithm for providing two steps: first PC and DTX and resource allocation for solved in optimization problem, second step for subcarrier allocation and power allocation at time variant in frequency selective channel. The inverse water filling algorithm is used for allocating the resource units in BS transmission. The relay [8]-[10] is used for cognitive radio network and it's mainly used for concurrent spectrum access network. The two slot protocol for bi-direction transmission and over all spectrum utilization. The Power allocation [11]-[13] for LTE Base Station in using Multiplexing technique for OFDM transmission scheme and different mechanisms are DTX, power control and antenna adaptation. The frame transmission for allocating resource are depends on time slot and subcarrier in user, inverse water filling algorithm used for allocation power.

The paper is systematized as follows: the system model is introduced at section I. In section II, proposed protocol for fixed D2D user acting as relay and power allocation for minimizing base station transmission power in described as numerical methods. In section III allocating power resource unit and algorithm implementation in relay selection and power allocation. Finally numerical result is discussed in section IV and section V for conclusion.

\section{SYSTEM MODEL AND RESOURCE ALLOCATION}

\subsection{Device to Device Communication}

The cellular Network for D2D Communication scenario is considered. Particularly consider Single-cell cellular network, consisting of BS and three users, denoted as D1, D2 and CU as shown in Fig: 1.The users D1 and D2 connected in local connections for e.g., data transfer, Base station allow two device communicate directly and each other in offload in traffic. In request D2D user to help BS to communicate in bidirectional way in another cellular user (CU). The spectrum sharing protocol model as bi-directional transmission between BS and D2D user, D2D communication, D2D and CU communication. 


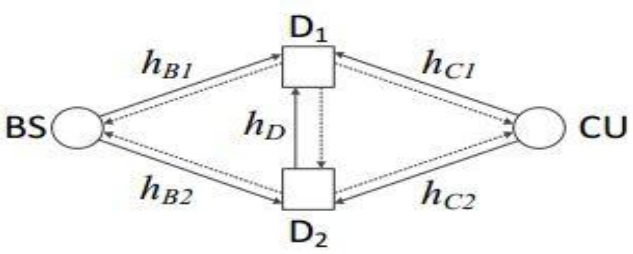

Fig: 1 D2D system model for proposed model

Proposed protocol considering following expression in Dn device signal to noise (SNR) is $\gamma_{n}$, D2D link achieved sum rate of D2D user as

$$
R_{D}=\frac{1}{2} \log _{2}\left(1+\gamma_{n 1}\right)+\frac{1}{2} \log _{2}\left(1+\gamma_{n 2}\right)(1)
$$

The allocating resource D2D users for one way or two way communication as,

$$
R=\max _{\beta_{B} \beta_{C} \in[0,1]} \frac{1}{2} \log _{2}(1+X)+\frac{1}{2} \log _{2}(1+Y)
$$

Subject to

$X=\frac{\beta_{c} \gamma C_{n}}{1+\frac{1}{\gamma_{n B}}\left(\beta_{B} \gamma B_{n}+\beta_{C} \gamma C_{n}+1\right)}$ and $X=\frac{\beta_{B} \gamma B_{n}}{1+\frac{1}{\gamma_{n C}}\left(\beta_{B} \gamma B_{n}+\beta_{C} \gamma C_{n}+1\right)}$

Finally equal optimization problem in resource allocation of user in D2D as

$$
R_{n n}^{(n)}\left(\beta_{B} \beta_{C}\right)+R_{n n}^{(n)}(\alpha)=R_{D}
$$

Subjected to

$$
\alpha, \beta_{B}, \beta_{C} \in[0,1]
$$

The resources are allocated for D2D communication for choosing value for next section in Results.

\subsection{Base Station}

The Downlink transmission one frame is consider to communicate between point-to-multipoint cellular networks, it consist of one Base station and multiple mobile terminals. The BS transmitter consisting number of MT antennas and every antenna share transmitting power.

The mobile receiver denoted as MR antennas, it shared resource via orthogonal frequency division multiple accesses (OFDMA) between the number subcarriers $(\mathrm{N})$, time slot $(\mathrm{T})$ and users $(\mathrm{K})$. The maximum resource for allocation is TN and OFDMA scheme for time variant channel to frequency selective in users.

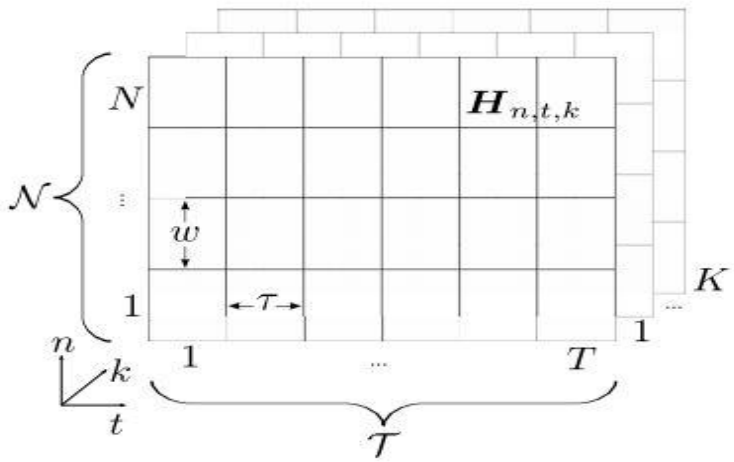

Fig: 2 OFDM frame model
The Resource unit considers the channel state matrix denoted as

$$
\mathrm{H}_{\text {n.t.k }} \in \mathrm{C}^{\mathrm{M}_{\mathrm{R}} * \mathrm{M}_{\mathrm{T}}}
$$

Where $\mathrm{n}=1, \ldots, \mathrm{N}$ (Subcarrier index), $\mathrm{t}=1, \ldots, \mathrm{T}$ (time slot) and $\mathrm{k}=1, \ldots, \mathrm{K}$, the power consumption model $\mathrm{BS}$ is as follows

$$
\mathrm{P}_{\text {supply }}\left(\mathrm{P}_{\mathrm{t}}\right)=\left\{\begin{array}{ll}
\mathrm{P}_{0, \mathrm{M}_{\mathrm{T}}}+\Delta_{\mathrm{PM}} \mathrm{P}_{\mathrm{t}} & \text { if } \mathrm{P}_{\mathrm{t}}>0 \\
\mathrm{P}_{\mathrm{S}} & \text { if } \mathrm{P}_{\mathrm{t}}=0
\end{array}(5)\right.
$$

The energy saving as follows

1. The Power control each Resource unit having reduce the transmission power

2. Antenna adaptation follows Minimizing the BS transmission power

3. Discontinues transmission follows increasing the time in BS and transmitting power in discontinuous.

Minimizing power supply consumption as follows in radio frequency transmission power,

$$
\mathrm{P}_{\mathrm{t}}=\sum_{\mathrm{a}=1}^{\left|\mathrm{A}_{\mathrm{t}}\right|} \sum_{\mathrm{e}=1}^{\left|\varepsilon_{\mathrm{a}}\right|} \mathrm{P}_{\mathrm{a}, \mathrm{e}}
$$

The power supply in transmission frame is as follows in different time slot and same frequency channel for allocating resource units.

$$
\mathrm{P}_{\text {supply , frame }}(\mathrm{r})=\frac{1}{\mathrm{~T}}\left(\sum_{\mathrm{t}=1}^{\mathrm{T}_{\text {Active }}}\left(\mathrm{P}_{0, \mathrm{M}_{\mathrm{T}}}+\Delta_{\mathrm{PM}} \mathrm{P}_{\mathrm{t}}\right)+\sum_{\mathrm{t}=1}^{\mathrm{T}_{\text {Sleep }}} \mathrm{P}_{\mathrm{S}}\right)
$$

Subject to $P_{t} \leq P_{\max }$ and $\mathrm{T}_{\text {Active }}+\mathrm{T}_{\text {Sleep }}=\mathrm{T}$

The power allocation in calculating sleep mode duration in Base station as follows

$$
T_{\text {Sleep }}=\frac{T N_{\mu K+1}-K}{N}
$$

Calculating remaining time slot is

$$
\mathrm{T}_{\text {Active }}=\mathrm{T}-\mathrm{T}_{\text {Sleep }}(9)
$$

Remaining unallocated resource and $m_{k}$ is assigned resource in users

$$
\mathrm{m}_{\mathrm{rem}}=\mathrm{NT}-\sum_{\mathrm{k}=1}^{\mathrm{K}} \mathrm{m}_{\mathrm{k}}-\mathrm{NT}_{\text {Sleep }}
$$

The allocating user $m_{k}$ is equal divided resource are time slot $\mathrm{m}_{\mathrm{k}, \mathrm{t}}$ with

$$
\mathrm{m}_{\mathrm{t}, \mathrm{rem}}=\left\lfloor\frac{\mathrm{m}_{\mathrm{k}}}{\sum_{\mathrm{t}=1}^{\mathrm{K}} \mathrm{m}_{\mathrm{l}}}\right\rfloor
$$

The remaining unassigned resource

$$
m_{t, r e m}=N-\sum_{k=1}^{K} m_{k, t}
$$


Time slot considered for discontinuous transmission for power control and antenna adaptation in resource allocation. The Result are analysis in section -IV. The equation (12) as un-allocated resource units.

\section{POWER ALLOCATION}

The power allocation for cellular networks transmission power in Singe-Input Multiple-Output (SIMO) and MIMO is processed for RAPS algorithm. The algorithm first to find number of user discontinuous transmission in sleep mode and allocating resource in depends on user, frequency, time. User rate of resource is allocated in inverse water filling algorithm. The unallocated resource is sharing spectrum in use of technologies is cognitive radio network.

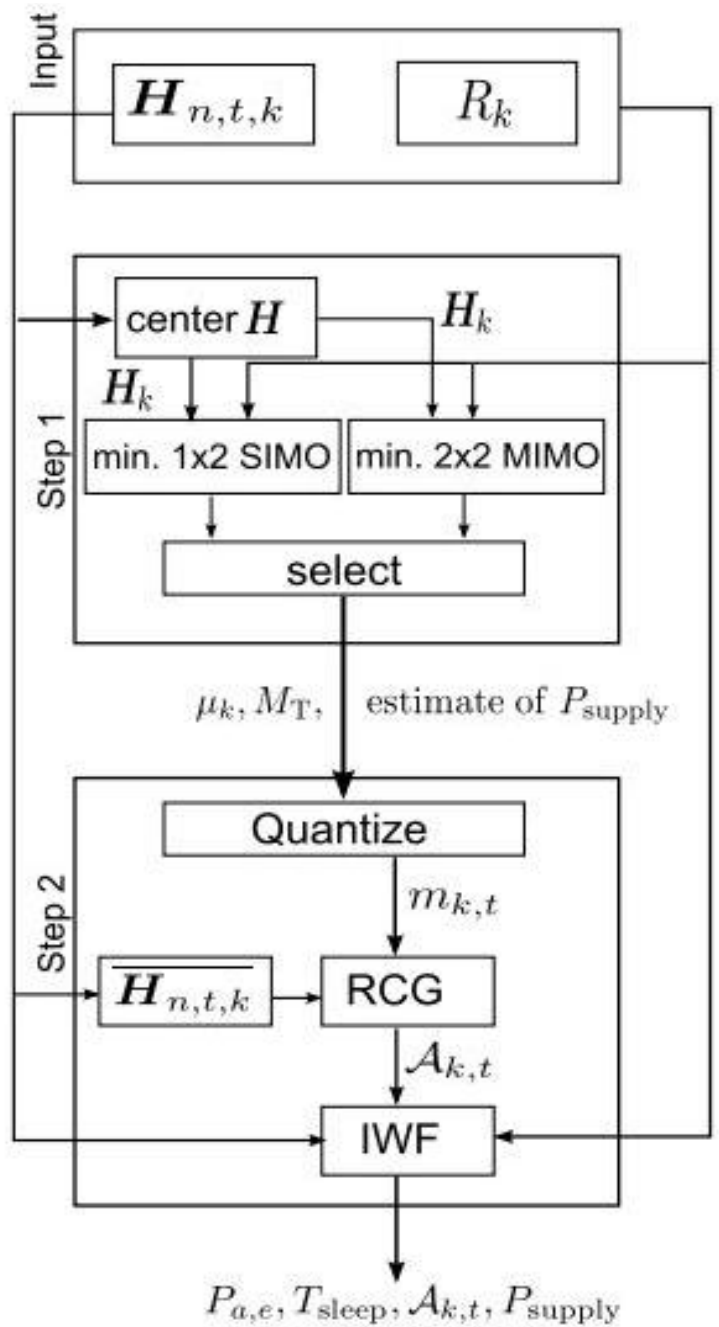

Fig: 3 Outline for RAPS algorithm

The Fig: 3 as power allocation cellular networks, first step to portioning the transmission frame and BS for finding allocated resource, sleep mode duration. To implementing the simulation is section IV. The result denotes for one-way and two-way communication for D2D user and CU user networks.

\section{RESULTS}

The performance of the RAPS algorithm simulations are configured as follows: BS and mobile terminal distance is $300 \mathrm{~m}$ minimum distance is $40 \mathrm{~m}$ to avoid SNRs. Fading is calculation as Non Line of sight channel with $8 \mathrm{~dB}$ shadowing signal. The simulation parameters as follows in Table 1 .
Table 1 Simulation Parameters

\begin{tabular}{|c|c|c|}
\hline Variable & & Value \\
\hline $\mathrm{K}$ & Number of user & 10 \\
\hline $\mathrm{N}$ & Number of subcarrier & 50 \\
\hline $\mathrm{T}$ & Number of time slot & 10 \\
\hline $\mathrm{M}_{\mathrm{T}}$ & $\begin{array}{c}\text { Number of transmitter } \\
\text { antenna }\end{array}$ & {$[1,2]$} \\
\hline $\mathrm{M}_{\mathrm{R}}$ & Number of receiver antenna & 2 \\
\hline $\mathrm{P}_{\max }$ & $\begin{array}{c}\text { Maximum transmission } \\
\text { power }\end{array}$ & $46 \mathrm{dBm}$ \\
\hline $\mathrm{N}_{0}$ & Noise spectral density & $4 \times 10^{-21} \mathrm{WHz}$ \\
\hline $\mathrm{P}_{0}, \mathrm{M}_{\mathrm{T}}$ & Circuit power consumption & $185 \mathrm{~W} / 260 \mathrm{~W}$ \\
\hline$\Delta_{P M}$ & Load dependence factor & 4.7 \\
\hline
\end{tabular}

The D2D communication for proposed protocol for relay selection in one way, two-way and selection is as follows. In this case $\mathrm{BS}$ to $\mathrm{D} 1$ is more stronger than $\mathrm{CU}$ to $\mathrm{D} 1$, interfernce is reduced for D2D user and also minimized transmission power.

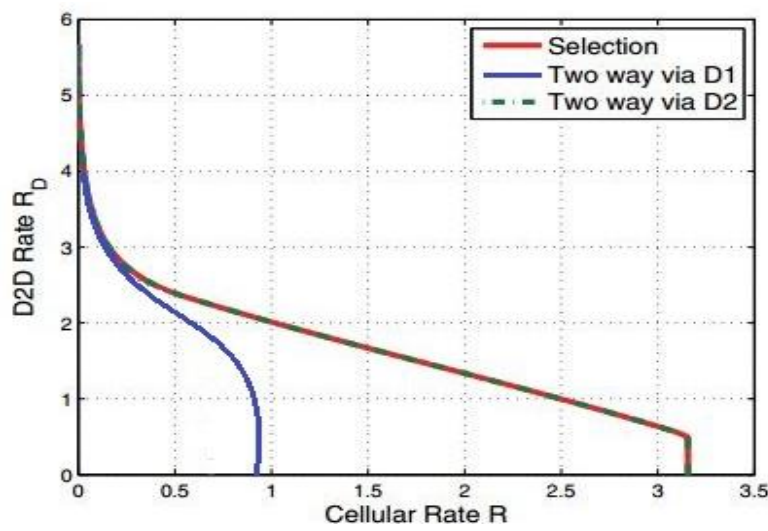

Fig: 4 Achievable rate regions with selection for D2D user

The proposed protocol for D2D user and D1 as fixed relay and first consider the two cellular network values are: $\mathrm{gB} 1=$ $\mathrm{gC} 1=0.2, \mathrm{gD}=0.6$. The relay selection is achieved highest cellular rate for D2D communications. The Base Station power consumption is follows in the offload traffic between BS and D2D users.

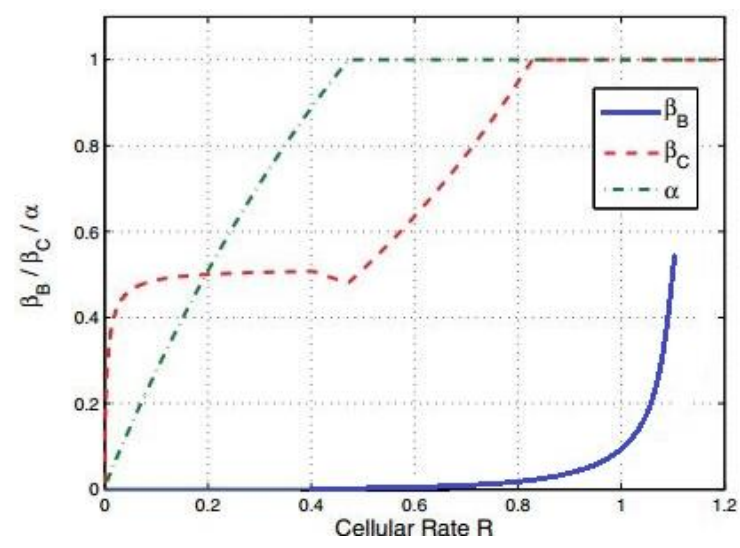

Fig: 5 The optimal $\left(\beta_{B}, \beta_{C}, \alpha\right)$ at resource allocation for proposed systems 
The cellular transmission for one way $\left(\beta_{C}=0\right.$ or $\beta_{B}=$ 0 or $\alpha=0$ )in fig: 5 , and comparing maximum power is $\beta_{B}, \beta_{C}=1$ applied to cellular network. The two way protocol for power control in cellular user is increasing the sum rate for cellular user and D2D transmission at fig: 4 .

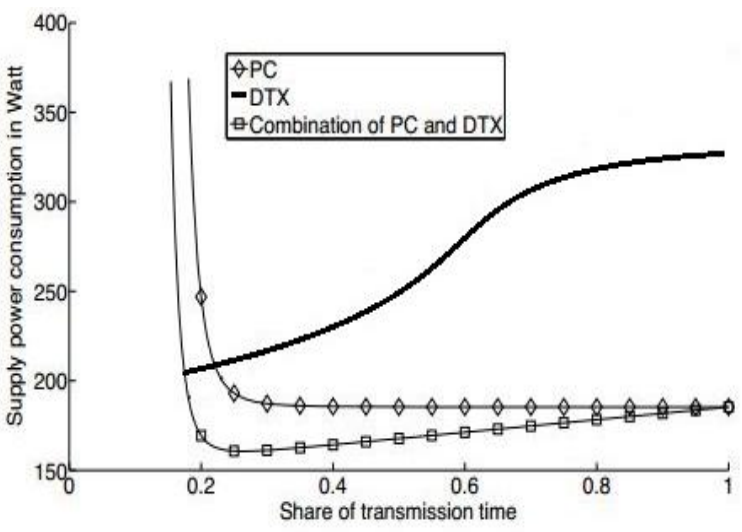

Fig: 6 Supply power consumption for transmission in three algorithms (PC, AA, DTX)

The power consumption model for the BS in three different algorithms and to implement power allocation in lower transmission power applied small BS. The same resource are allocated for frequency selective and time variant channel allocation in cellular network. The algorithm is denotes for working of relay network and transmission model different multiplexing.

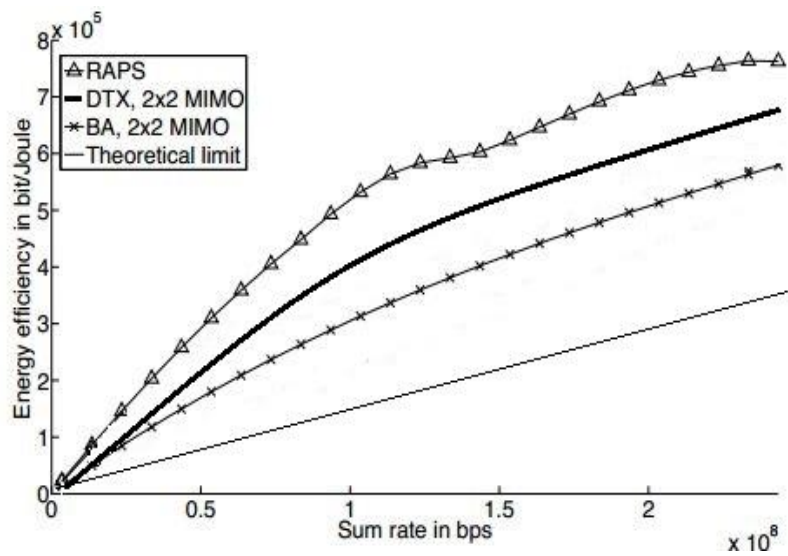

Fig: 7 Energy efficiency in transmission scheme

The RAPS can be applied to many of BSs, the simulation results are mentioned above fig:7 in this figure comparing all parameters RAPS achieved higher power consumption in cellular networks.

\section{SUMMARY AND CONCLUSION}

In this paper, we proposed a spectrum sharing protocol for D2D communication and BS power saving mechanism provides power control, antenna adaptation and Discontinuous transmission. That protocol provides bi-directionally two-way communication between D2D users and BS, CU. The RAPS algorithm provides mechanism for minimizing the power consumption, first an estimate the resource units and second step allocating power resources in cellular users. The result proposed two-way protocol with power control in D2D communication and cellular user communication. The future work to RAPS in minimizing multi cell interference and increasing high speed communication between the D2D for ad-hoc networks.

\section{REFERENCE}

[1] Y. Pei and Y. C Liang, "Resource allocation for device to device communications overlaying two-way networks", IEEE Trans on Wireless Comm, vol. 12, pp 3611-3621, 2013.

[2] P. Phunchangharn, E. Hossain, and D. I. Kim, "Resource allocation for device to communications underlaying LTE -advanced networks," IEEE trans on Wireless Comm , vol. 20, no. 4, pp.91-100, 2013.

[3] B. Kaufman ,J. Lileberg and B. Aazhang, "Spectrum Sharing Scheme Between cellular User and Ad-hoc Device to Device User," IEEE Trans on WirelssComm, vol. 12, no.3, pp. 1038-1049, March 2013.

[4] L. Lei, T. Zhang, X. Shan, C. Lin and Z. Zhong, "Performance Analysis of Device to Device Communication with Dynamic Interference Using Stochastic Petri Nets,"in IEEE Trans on Wireless Comm, vol.12, no. 12,pp. 6121-6141, 2013.

[5] HaukeHoltkamp, Gunther Auer, SamerBazzi and Harald Hass, "Minimizing Base Station Power Consumption", in IEEE journal on Selected areas in Comm, vol. 32, no. 2, pp.291-306 Feb-2014.

[6] L. Lei, Z. Zhong, C. Lin and X. Shen, "Operator Controlled Device to Device communication in LTE advanced network," IEEE Wireless comm , vol. 19, no. 3, pp. 96-104, june 2012.

[7] H. Min , W.Sao, J. Lee, S. Park and D. Hong, "Reliability Improvement Using Receive mode Selection in the Device to Device Uplink period Underlaying Cellular Networks," in IEEE Trans on Wireless Comm, vol. 10, no. 2, pp. 413-418. Feb-2011.

[8] P. Janis, C. H. Yu, K. Doppler, C. B. Ribeiro, C. Wijting, K. Hugl, O. Tirkkonen and V. Koivunen, Device to Device Communication underlaying cellular communication systems, "Int. J. Comm Network and system sciences, vol. 2, no. 3, pp. 169-178, 2009.

[9] C. H. Yu, K. Doppler, C. B. Ribeiro and O. Tirkkonen, "Resource sharing Optimization for device to device communication underlaying cellular networks, "IEEE trans. Wireless comm, vol. 10, no. 8, pp. 2752-2763, Aug-2011.

[10] M. Hedayati, M. Amirijoo, P.Frenger and J. Moe, "Reducing Energy Consumption through Adaptation of Number of Active Radio Units," in proc. IEEE VTC 2012 -spring, 2012.

[11] C. Dessset, B. Debaillie, V. Giannini, A. Fehske, G.Auer, H. Holtkamp,W. Wajda, D.Sabella, F. Richter, M. Gonzalez, H. Klessig, I. G'odor, P. Skillermark, M. Olsson M. A. Imran, A. Ambrosy and O. Blume, "Flexible power modeling of LTE base stations," in IEEE Wireless communications and networking conference, in 2012.

[12] R. Wang, J.Thompson, H. Haas and P. Grant, "Sleep Mode Design for Green Base Stations," IET communications, vol. 5, no. 18, pp. 2606-2616, 2011.

[13] H. Al-Shatri and T. Weber, "Fair Power Allocation for Sum-Rate Maximization in Multiuser OFDMA," in Proc. International ITG Workshop on Smart Antennas, 2010 